Meta

Journal des traducteurs

Translators' Journal

\title{
Traduction et industries de la langue : nouveau défi pour le traducteur?
}

\section{Jean-Claude Gémar}

Volume 37, numéro 2, juin 1992

URI : https://id.erudit.org/iderudit/002874ar

DOI : https://doi.org/10.7202/002874ar

Aller au sommaire du numéro

Éditeur(s)

Les Presses de l'Université de Montréal

ISSN

0026-0452 (imprimé)

1492-1421 (numérique)

Découvrir la revue

Citer cet article

Gémar, J.-C. (1992). Traduction et industries de la langue : nouveau défi pour le traducteur ? Meta, 37(2), 374-378. https://doi.org/10.7202/002874ar d'utilisation que vous pouvez consulter en ligne.

https://apropos.erudit.org/fr/usagers/politique-dutilisation/ 


\section{TRADUCTION ET INDUSTRIES DE LA LANGUE : NOUVEAU DÉFI POUR LE TRADUCTEUR ?1}

Je commencerai par une citation, extraite de L'Homme de paroles, de Claude Hagège:

Ceux qui voudraient lui dénier [à la traduction] toute valeur de critère, sous prétexte qu'on traduit toujours misérablement, doivent bien admettre que tout texte d'une langue - car ce sont des textes, et non des langues, que l'on traduit - est, approximativement ou parfaitement, traduisible en un texte d'une autre. (Paris, Fayard, 1985, p. 47.)

Un peu plus loin, Hagège ajoute ceci :

Mais le plus étonnant est que même imparfaitement, même de façon très approximative, on puisse toujours traduire (p. 50).

Tout le mystère de la traduction tient dans ces quelques mots.

Lorsque Yahweh, dans sa colère, condamna à l'échec, faute de pouvoir communiquer entre eux, les imprudents qui osaient le défier (Genèse, 11), il n'avait pas imaginé que l'homme trouverait un jour la parade. La traduction est en effet la réponse des descendants d'Adam et d'Ève à la malédiction divine. Très imparfaite souvent, fragile toujours, voire approximative, la traduction est une tentative - plus ou moins réussie selon les genres, les lieux et les époques - obstinée et itérative de 1'horro sapiens sapiens en vue de communiquer toujours et partout avec ses semblables en dépit des difficultés, des obstacles ct des risques que présente l'acte dialogal quand il chevauche deux langues.

Le développement inouï des communications auquel nous assistons depuis la fin de la Deuxième Guerre mondiale a propulsé la traduction (orale et écrite) au rang de discipline universitaire à part entière enseignée dans un grand nombre de pays. Si l'on peut épiloguer sans fin sur la nature de la traduction (est-ce un art, une science, un savoirfaire ou une technique?), on ne mettra pas en doute pour autant qu'elle s'enseigne et qu'elle s'apprend, car elle répond à un besoin social reconnu par l'Université, parfois depuis des temps très anciens. Ce besoin, quoique très réel, n'est pas toujours (ou encore) ressenti de façon aussi aiguë ou nécessaire dans les pays de l'hémisphère sud que dans ceux du nord. Il a donc donné naissance, chez ces derniers, à une tradition solide, étayée par des décennies d'expérience et de pratique, nationale et internationale, fondée sur une langue véhiculaire ayant parfois le statut de lingua franca.

Cette expérience, cette pratique, les pays du nord qui les possèdent ont le devoir de les communiquer aux pays démunis, non pour qu'ils les reprennent à leur compte tels quels sans aménagement ni adaptation, à l'instar de certains modèles occidentaux importés directement sans distanciation aucune, mais pour qu'ils gagnent un temps précieux dans le processus de réflexion et d'analyse préalable à la mise en cuvre de programmes d'enseignement de la traduction et de ses disciplines associées.

Qui dit enseignement sous-entend pédagogie, autrement dit manière d'enseigner (ou conception, éthique de l'enseignement) en fonction de critères particuliers, propres à un pays, à sa culture et à la langue qui la véhicule. À cet égard, avant de songer à appliquer des «méthodes de traduction», il faut s'interroger longuement et profondément sur le pourquoi et le comment de celle-ci. Une fois les objectifs définis, l'objet même de la traduction $s$ 'en trouvera considérablement éclairé. Il restera ensuite à ajuster les moyens aux buts visés.

Mais, me direz-vous, que viennent faire les «industries de la langue» dans l'univers du traducteur? En quoi le concernent-elles ? Et pour commencer, qu'est-ce que ce terme recouvre réellement? Afin de clarifier un peu les choses, je vous répondrai ce que mon collègue Jean Baudot et moi-même en avons dit dans la revue Universités (mars 1988, pp. 25-26):

Apparue voici une trentaine d'années, l'informatique a mis plus de vingt ans avant d'offrir des outils de travail répondant aux besoins des personnes cuvrant dans le domaine langagier: traducteurs, rédacteurs, lexicographes, terminologues et autres artisans de la langue. 
Ce retard a été comblé, au point que depuis une décennie le marché des logiciels et des progiciels est inondé de systèmes. En voici quelques exemples :

- traitement de textes

- production de relevés de vocabulaire ;

- production d'index;

- génération de concordances;

- vérification de l'orthographe;

- courrier électronique

- aide à la traduction;

- bases de données plus ou moins adaptables à des données lexicographiques ou terminologiques;

- édition électronique, etc.

La plupart de ces logiciels ont été d'abord conçus pour le traitement de la langue anglaise et n'étaient pas immédiatement applicables à la langue française; il fallut soit les adapter, soit en faire de nouveaux pour répondre aux particularités morphosyntaxiques du français. C'est ainsi que prirent naissance les «industries de la langue», du moins celles qui s'appliquent au domaine français.

Bien que le terme «industries (informatiques) de la langue» et les notions qu'il recouvre aient été longs à prendre forme, en raison surtout des difficultés de traitement des données linguistiques, cette nouvelle forme d'activité de production (de services) ne cesse de croître en importance et s'annonce capitale pour l'avenir du français et des langues en général, notamment pour les quatre raisons suivantes:

1. Une évidence s'impose désormais : la machine à écrire a vécu, elle deviendra rapidement un objet de musée. Elle est déjà largement remplacée par les systèmes de traitement de texte sur ordinateurs individuels. Ceux-ci, à leur tour, sont appelés à faire partie de stations de travail «bureautiquisées», dont le traitement de texte ne constituera qu'une des nombreuses ressources disponibles.

2. Une grande partie des documents imprimés sur papier sera bientôt remplacée, pour les consommateurs, en aval, par des enregistrements sur de nouveaux supports :

- réels (disquettes, disques compacts, disques optiques de très grande capacité) ou

- virtuels (banques de donnés centralisées, réseaux de télématique).

- Dans la première catégorie figureront lexiques, dictionnaires, terminologies multilingues, encyclopédies, ouvrages de référence, livres dits «classiques», livres contemporains.

- Dans la deuxième, on peut s'attendre à voir journaux, magazines et périodiques; bottins, annuaires, horaires, etc.; publicités commerciales; reférences documentaires, bibliothèques spécialisées, photothèques.
Les industries de la langue seront appelées à collaborer non seulement à la production de tels documents mais encore à la fabrication d'outils de «consommation» (entendre: consultation) de ces documents.

3. Un tout autre type de recueils lexicographiques et terminologiques devra être mis au point, non plus pour des consommateurs humains, mais pour répondre aux exigences de plus en plus linguistiques des systèmes d'intelligence artificielle. Il s'agit ici de véritables dictionnaires électroniques répertoriant les diverses unités de la langue, accompagnées (au minimum) de renseignements détaillés sur leur comportement morphologique, syntaxique et sémantique.

- Une telle entreprise exigera de nombreuses années de travail d'équipes d'ingénieurs-linguistes, travail toujours à reprendre et à perfectionner pour mieux couvrir la réalité linguistique et suivre l'évolution des langues techniques de spécialité.

4. Depuis ELIZA ${ }^{2}$, le dialogue «hommemachine» a fait des progrès, mais nous sommes encore loin de pouvoir converser en langue naturelle, sans restrictions, avec les ordinateurs. Quant au dialogue en langue parlée, l'entreprise s'avère encore plus complexe, car la conversation suppose résolus deux problèmes difficiles:

$1^{\circ}$ la compréhension d'un message, à l'aide

- de dictionnaires électroniques ;

- d'une maîtrise de la grammaire de la langue;

- d'un accès à un minimum de connaissances accumulées;

$2^{\circ}$ la formation d'un message à l'aide

- des ressources énoncées en $1^{\circ}$;

- d'un système intelligent qui engendre le contenu du message.

Avant d'espérer obtenir des progrès significatifs dans cette direction, il faudra prévoir, quoi qu'on dise, quelques décennies de travail. Il reste que l'avenir des industries de la langue et de ses artisans est prometteur. La formation de ces «travailleurs» d'un nouveau genre pose toutefois un nouveau défi aux universités et aux pédagogues responsables des questions langagières, celui de prévoir de nouveaux outils pédagogiques en fonction des besoins, aussi divers que nombreux, semble-t-il, des consommateurs de demain.

Après ce petit détour dans le futur proche, revenons à la traduction et à ses origines.

Activité aussi vieille que l'humanité (5000 ans attestés), la traduction est essentiellement «humaine» jusque dans les années 1960 . On considérait alors sa manifestation la plus visible et connue, la traduction littéraire, comme un art.

Depuis le développement de l'informatique, la traduction est de moins en moins le seul fait de 
l'homme et de plus en plus celui de la science et de la technique. Elle est notamment une activité moins autonome, puisque sa réalisation est plus ou moins étroitement liée, selon le domaine d'application, entre autres à l'utilisation des moyens et techniques suivants :

- traitement de texte;

- traduction assistée par ordinateur (TAO);

- traitement automatique de l'information;

- banques et banquettes de terminologie;

ainsi qu'aux progrès de l'intelligence dite artificielle.

Il s'ensuit que la nature même de la traduction a changé. On attend du traducteur davantage un savoir-faire qu'une simple compétence, La part des textes «pragmatiques» augmente, car ils sont le produit des innombrables activités humaines des sociétés actuelles, assoiffées de connaissances mais pas nécessairement de savoir - et dévoreuses d'informations en tous genres. Proportionnellement, la place de la traduction traditionnelle, celle des textes esthétiques, ne cesse de diminuer au profit de la traduction utilitaire, de nature strictement commerciale, économique ou politique.

Qu'il le veuille ou non, qu'il le déplore ou s'en réjouisse, le traducteur est bien obligé de suivre cette évolution. Le «pédagotrad» (ou professeur de traduction) aussi.

\section{LA TRADUCTION COMME ACTE DE FOI}

Si l'opération de traduction est bien, selon les théoriciens du langage, un acte de communication avant tout, elle est au moins autant un «acte de foi»: foi dans la réussite de la communication interlinguistique recherchée. Le but premier du traducteur est de réussir à :

1. comprendre le message;

2. le rendre;

3. le faire passer.

Pour ce faire, la machine ou la technique ne représente qu'un moyen plus ou moins efficace de faire passer la communication. Le traducteur reste irremplaçable, du moins pour le moment. C'est la machine qui est (encore) au service de l'Homme, et non le contraire.

Aussi importe-t-il de former le traducteur sur le double plan de la compétence et de la performance (ou savoir-faire). En premier lieu, le traducteur doit avoir atteint un niveau de compétence suffisant dans la langue source pour comprendre le texte de départ (TD) le mieux possible. Ensuite, le niveau de son savoir-faire doit être au moins égal pour produire en langue cible un texte «équivalent» (je n'ai pas dit «identique»!) au TD.

Le traducteur a donc une double (ou quadruple) obligation, à la fois vis-à-vis des langues source et cible et à l'égard des textes de départ et d'arrivée.

\section{RESPONSABILITÉS ET FORMATION DU TRADUCTEUR. LA PARTIE...}

La responsabilité du traducteur devant la société est grande. Elle est plus ou moins lourde selon le type de texte en cause, selon qu'il sera porteur ou non d'enjeux engageant la totalité ou une partie de la collectivité, un groupe ou une personne seulement. C'est toute la différence que l'on peut établir entre un texte fondamental, à vocation universelle, comme la Déclaration universelle des droits de l'homme et du citoyen, une convention collective donnée et le mode d'emploi d'un aspirateur.

$\mathrm{La}$ formation du traducteur doit aussi le préparer à assumer cette responsabilité, qui ne sera pas moins contraignante en l'an 2000 qu'elle ne l'était au XIxe siècle, en 1918 ou en 1985, parce que le texte sera affiché sur un écran cathodique plutôt que rédigé sur un support de papier.

La formation actuelle du traducteur repose sur un certain nombre de principes, dont voici l'essentiel:

1. connaissance des langues (départ et arrivée);

2. maittrise des outils documentaires (la documentation);

3. culture générale développée;

4. formation en terminologie générale et, le cas échéant, dans un domaine (et ses modes d'expression).

Le traducteur de l'an 2000, lui, sera tenu d'avoir une :

1. connaissance poussée des langues $(3,4,5$, et non 1 ou 2 seulement!);

2. maîtrise des machines et des moyens informatiques (dont celle des banques de données et dictionnaires électroniques);

3. formation approfondie en terminologie /linguistique générale et appliquée, cela pour répondre aux nouveaux besoins du marché;

4. vaste culture générale, diversifiée, scientifique et littéraire comprises.

D'après ce profil de formation, la responsabilité du professeur de traduction, le formateur, est également double:

10 Il doit enseigner un savoir-faire, donner au traducteur le degré de compétence suffisant pour en faire un bon «travailleur de la langue» possédant à fond les bases de son métier, notamment à partir de l'emploi plus ou moins généralisé de la machine.

C'est l'obligation absolue du formateur, celle que 1'on est en droit d'attendre d'une personne qui prépare à un métier, qu'elle forme des ingénieurs, des comptables ou des dentistes. Mais le formateur doit en outre:

$2^{\circ}$ enseigner aux futurs traducteurs un savoirêtre, celui qu'ils devront maîtriser pour vivre dans le $\mathrm{XXI}^{\circ}$ siecle, où l'on verra se répandre à peu près partout le fruit du progrès de la technique, soit les systèmes informatiques et la TAO. 
L'obligation du formateur, en l'occurrence, n'est que relative - car fonction du contexte socio-économique, notamment, dans lequel il enseigne - mais les incidences n'en seront que plus graves, selon qu'il aura pris en compte ou non le facteur humain.

L'enjeu, ici, est d'ordre personnel (c'est l'individu qui est en cause), mais le coût social qu'il entraînera n'en sera pas moindre pour autant.

\section{... ET LE TOUT :}

\section{LA RESPONSABILITÉ COLLECTIVE}

Le futur travailleur des industries de la langue du $\mathrm{XXI}^{\mathrm{e}}$ siècle - et ce terme englobe traducteurs, interprètes, terminologues, rédacteurs, réviseurs, et j'en passe - est appelé à œuvrer dans des conditions qui seront sans doute plus faciles sur le plan matériel, technique, grâce au support informatique, mais qui n'en seront pas moins plus difficiles sur le plan humain, personnel. En effet, les conditions de travail favoriseront l'isolement de l'individu, exigeront un niveau d'attention élevé; de là une fatigue accrue, des risques plus grands d'erreur, etc.

En outre, l'addition de la technique et de l'information, c'est-à-dire le traitement automatique des «données» (les célèbres data!), ne donne pas nécessairement pour résultat une augmentation du savoir, un progrès de la pensée ni, a fortiori, un savoir éclairé dont profiterait le traducteur, mais plutôt des éléments, des fragments de connaissances qu'il lui appartient d'exploiter optimalement. Autrement dit, il reste le maître du jeu.

Il faut donc se garder de voir dans le progrès que représentent les machines et l'électronique la solution à tous nos problèmes de civilisation. $\mathrm{La}$ traduction, ni moins ni davantage que les autres activités humaines fondées sur le fonctionnement de l'esprit, n'est pas près de se faire toute seule. Les banques de données n'ont pas rendu désuets les dictionnaires conventionnels, elles ne se substituent pas à la pensée et au jugement, et encore moins à l'intelligence: elles ne sont que la manifestation, spectaculaire certes, de son savoirfaire, pas de son savoir-être. Or, c'est ce savoirêtre qui doit être développé, car, si l'on en croit les propos du cardinal Lustiger: «Le véritable problème contemporain [...] c'est celui de la maîtrise que donne sur la condition humaine la connaissance scientifique et technique ${ }^{3}$.»

Si les paroles du cardinal évêque de Paris sont justes, il faudra alors que l'humanité possède «un surcroît de sagesse pour que le coût humain [de cette maîtrise] ne soit pas aussi féroce que celui de l'évolution économique et industrielle ${ }^{2} \gg$, de triste mémoire.

Encore une fois, la réponse nous est peut-être donnée par Octavio Paz:
Le grand secret de notre époque est sans doute de trouver le point où le principe de changement se confond avec celui de permanence. Pour qu'entre le passé surpeuplé et le futur inhabité advienne enfin le présent. (Dans un entretien accordé à $L^{\prime}$ Express, $\mathrm{n}^{\circ}$ 1933, juillet 1988, p. 50.)

C'est en effet en tirant les leçons du passé, et surtout du passé récent, que nous préparerons le terrain sur lequel cuvrera le traducteur de demain. Entre un passé lointain et un avenir incertain, il importe de repenser le présent, et tout particulièrement le concept de culture, francophone en l'occurrence. Je veux dire par là qu'il importe d'instaurer une culture francophone qui soit le reflet de son temps. Pour cela, je vous propose une réévaluation de la notion traditionnelle de culture.

\section{POUR UNE RÉEVALUATION DE LA NOTION DE CULTURE}

Je crois que toute future définition de la culture devrait prendre en compte les deux facteurs essentiels que sont d'une part l'évolution irrésistible des sociétés et des mours, au nord comme au sud, depuis une génération (1968?), et celui, corollaire, de la notion de «langue» dans le contexte d'une civilisation de plus en plus médiatique et vouée, pour le meilleur ou pour le pire, à la communication.

Les fondements de la culture traditionnelle ont été ébranlés en profondeur par les bouleversements qu'a subis la société depuis une génération. Ce fait est indéniable. Il est observable au nord comme au sud. Le nier reviendrait à nier l'évidence. Il s'ensuit une transformation, subtile et pas toujours nette, de la «culture» (qui est, selon Alain Touraine, la manière dont une collectivité construit ses relations avec son environnement).

Il semble bien que nous nous acheminions vers une forme mixte, davantage intégrée de la culture. D'ores et déjà, elle ne repose plus totalement sur le seul pilier de la littérature, sauf chez quelques clercs appartenant à une espèce révolue. Le «patrimoine informationnel» de l'honnête homme de notre temps ne peut - ne doit - plus être constitué de la seule connaissance des mœurs et œuvres littéraires d'une époque. Désormais, comme au XVIII ${ }^{\mathrm{e}}$ siècle, il doit s'appuyer aussi, sinon davantage, sur la connaissance des faits, événements sociaux, économiques, politiques, scientifiques et techniques de notre temps. C'est ainsi que se forme une «culture générale», qui devrait être composée autant des savoirs que des savoirfaire d'une société.

\section{LA LANGUE AU SERVICE DE LA SOCIÉTÉ, ET NON L'INVERSE}

Cette transformation de la société contemporaine passe bien évidemment par le canal de la 
langue, ce qui pour nous signifie que la langue (française en l'occurrence) doit s'adapter à cette évolution, et non l'évolution être orientée en fonction de la langue.

Les aptitudes et qualités de notre langue ne sont pas en cause. D'ailleurs, qui pourrait douter de sa capacité à suivre le train du développement, de la science et de la technique, quand on voit la facilité avec laquelle elle se coule dans les mots et concepts nouveaux que forge l'anglais quotidiennement? Les résultats plus que probants de la terminologie (unilingue et bilingue) parlent d'euxmêmes. Non, c'est véritablement sur le plan des mentalités, vis-à-vis de la notion traditionnelle de langue, perçue comme le véhicule quasi sacré d'une vérité immuable, que nous devons faire porter nos efforts.

À cet égard, un constat s'impose: la langue française véhicule de plus en plus une culture dérogeant aux mythes et valeurs traditionnels de légèreté, de qualité de vie et de douceur de vivre quand elle projette l'image d'une nation commerciale, d'une puissance économique, militaire et industrielle majeure, et non plus seulement celle d'un pays voué au culte de l'intellect, de la belle et bonne langue et... de la mode, fût-elle intellectuelle.

Ce constat se traduit par des besoins nouveaux, ceux d'une langue française exprimant aussi clairement et simplement que l'anglais, par exemple, les notions liées à ces réalités scientifiques, techniques, commerciales, etc. La langue française n'est pas seulement la langue de Molière, elle est aussi, et de plus en plus nettement, celle de l'aérospatiale, du TGV, de la médecine, des télécommunications, entre autres.

Il s'ensuit que la notion de langue française doit s'inscrire profondément dans le cadre de la culture dont elle est désormais porteuse et que le principe des «études françaises» doit être revu en conséquence. De même pour son enseignement, qui devra en être le reflet. Il portera donc de plus en plus sur les matières et disciplines nouvelles, comme y invitent notamment les «industries de la langue» et les langues de spécialité (scientifique et technique, juridique, médicale, etc.), les disciplines linguistiques et leurs applications.

C'est à cette condition que l'on renouvellera l'enseignement de la langue française, langue de son temps, et que les futurs enseignants de français, langue première ou seconde, revaloriseront leur statut professionnel (et scientifique) aux yeux de la société. Mais, hélas, cela ne se fera pas tout seul: ils devront tout d'abord s'en convaincre, puis se mettre sérieusement au travail. Le veulent-ils? Le peuvent-ils? C'est une autre question, mais la poser revient à y répondre... Une chose me paraît sûre, en tout cas: si les professeurs de français et ceux qui les forment ne réagissent pas très rapidement - dans les pays du Nord comme dans ceux du Sud - pour prendre les mesures difficiles mais nécessaires qui s'imposent, ils s'exposent non seulement à tous les dangers qu'engendrent l'apathie générale et le découragement que l'on peut observer urbi et orbi devant les progrès qu'accomplissent les autres langues et qui font reculer le français et son enseignement, mais, plus grave encore, ils compromettent son avenir et celui de leurs futurs collègues.

Pour conclure mon propos, je voudrais proposer à votre réflexion une autre citation, celle d'un poète encore, Kenneth White :

[...] voilà ce que peut faire une traduction: relier des cultures étrangères l'une à l'autre, et préparer le terrain d'un tertium quid, un nouveau monde intellectuel. (Une apocalypse tranquille, Paris, Grasset, 1985, p. 52.)

Ce tertium quid, n'est-ce pas là, en fin de compte, le but que vise le traducteur, ce «messager de l'esprit», selon le joli mot de Pouchkine? À en croire Isaac Bashevis Singer, étant donné que chaque langue possède ses propres vérités uniques, la traduction est «'essence même de la civilisation». C'est cette essence qu'il nous faut retrouver, ne serait-ce que pour faire pièce à la malédiction de Babel. Et puis, n'oublions pas, comme nous le rappelait fréquemment Borges, le bibliothécaire de Babel, que «les mots ne sont pas seulement un moyen de communication mais aussi des symboles magiques et de la musique».

\section{JEAN-Claude GÉmar Université de Montréal, Montréal, Canada}

Notes

1. Communication présentée aux SEDIFRALE 7 (Belo Horizonte, Brésil) dans le cadre de la table ronde «Langues, sciences et technique», juillet 1989.

2. ELIZA est un des premiers systèmes (développé par J. Weizenbaum au MIT en 1966) qui répondait à des remarques qui lui étaient soumises au clavier d'une console.

3. Jean-Marie Lustiger, cardinal évêque de Paris. Propos extraits du livre d'entretiens avec Missika et Wolton: Le Choix de Dieu, Paris, 1987, Éditions de Fallois. 